\title{
Coherent patterning of matter waves with subwavelength localization
}

\author{
J. Mompart, ${ }^{1}$ V. Ahufinger, ${ }^{1,2}$ and G. Birkl ${ }^{3}$ \\ ${ }^{1}$ Departament de Física, Universitat Autònoma de Barcelona, 08193 Bellaterra, Spain \\ ${ }^{2}$ Institució Catalana de Recerca i Estudis Avançats (ICREA) Lluís Companys 23, 08010 Barcelona, Spain \\ ${ }^{3}$ Institut für Angewandte Physik, Technische Universität Darmstadt, Schlossgartenstraße 7, 64289 Darmstadt, Germany
}

(Received 17 November 2008; published 28 May 2009)

\begin{abstract}
We propose the subwavelength localization via adiabatic passage (SLAP) technique to coherently achieve state-selective patterning of matter waves well beyond the diffraction limit. The SLAP technique consists in coupling two partially overlapping and spatially structured laser fields to three internal levels of the matter wave yielding state-selective localization at those positions where the adiabatic passage process does not occur. We show that by means of this technique matter wave localization down to the single nanometer scale can be achieved. We analyze in detail the potential implementation of the SLAP technique for nanolithography with an atomic beam of metastable $\mathrm{Ne}^{*}$ and for coherent patterning of a two-component ${ }^{87} \mathrm{Rb}$ Bose-Einstein condensate.
\end{abstract}

DOI: 10.1103/PhysRevA.79.053638

PACS number(s): 03.75.Nt, 42.50.St, 42.50.Gy, 42.82.Cr

\section{INTRODUCTION}

The highly controlled manipulation of atomic matter waves has proven to be an exciting field of research in recent years. Specially, research in Bose-Einstein condensation, Fermi quantum degeneracy, and quantum information processing with ultracold atoms has achieved tremendous advances [1]. Future progress in this field will strongly profit from optical addressability, localization, and patterning of atomic systems with a resolution not limited by the wavelength of the radiation involved. Some important examples are site-specific addressing of ultracold atoms in optical lattices [2], patterning of Bose-Einstein condensates (BECs) [3], and atom lithography [4] based on light forces [5], optical quenching [6], or multiphoton processes [7].

Recently, there have been several proposals for subwavelength atom localization based on the interaction of threelevel atoms with light having a space-dependent amplitude distribution, mainly standing wave (SW) fields [8-13]. In all these proposals a spatially modulated dark state is created by means of either electromagnetically induced transparency (EIT) or coherent population trapping (CPT) [14]. In fact, a proof-of-principle experiment based on the CPT technique reported intensity patterns in the transmission of a probe field presenting subwavelength spatial resolution [15]. Significant for the present work, the CPT technique with a SW control field produces atom localization in one of the ground states with a spatial fringe pattern resembling that of a Fabry-Pérot resonator with cavity finesse given by the ratio $\mathcal{R}$ between the control and probe field intensities [11].

In this paper, we propose a state-selective atom localization and patterning scheme based on stimulated Raman adiabatic passage (STIRAP) $[16,17]$ that, compared to the CPT based techniques, presents several important advantages: (i) it produces "superlocalization," i.e., narrower localization than that expected from the CPT-finesse parameter $\mathcal{R}$; (ii) it is a fully coherent process that does not rely on spontaneous emission to the dark state and, therefore, it can be applied to open three-level systems and to systems where coherence has to be preserved such as BECs; (iii) the localized state does not suffer from recoil induced broadening and, therefore, the Raman-Nath approximation holds [18], and, finally, (iv) it is robust under uncontrolled variations in the system parameters, e.g., intensity fluctuations of the laser fields. We describe here the main features of this subwavelength localization via adiabatic passage (SLAP) technique, as well as its potential implementation for matter wave lithography down to the single nanometer scale and for coherent patterning of a BEC at the Heisenberg limit. Note that STIRAP without the spatial localization feature introduced here has been proposed [19] and recently experimentally demonstrated [20] for the transition from an atomic to a molecular BEC and for the optical control of the internal and external angular momenta of an extended BEC [21].

The paper is organized as follows. In Sec. II we describe the basics of the SLAP technique and derive semianalytical conditions for achieving the superlocalization regime. In Secs. III and IV we discuss the application of the SLAP technique for nanolithography with a $\mathrm{Ne}^{*}$ atomic beam and for coherent patterning of a two-component ${ }^{87} \mathrm{Rb} \mathrm{BEC}$, respectively. In Sec. V we further comment on other possible applications of the SLAP technique and present a short conclusion.

\section{SLAP TECHNIQUE}

The schematics of the SLAP technique are shown in Fig. 1. A plane matter wave formed by three-level atoms in a $\Lambda$-type configuration propagates at a velocity $v_{z}$ through two partially overlapping laser fields: the traveling wave (TW) couples the $|c\rangle \leftrightarrow|b\rangle$ transition with a Rabi frequency $\Omega_{\mathrm{TW}}(t)=\Omega_{\mathrm{TW} 0} \exp \left[-\left(t-t_{\mathrm{TW}}\right)^{2} / \sigma_{\mathrm{TW}}^{2}\right]$ and the SW couples the $|c\rangle \leftrightarrow|a\rangle$ transition with a Rabi frequency $\Omega_{\mathrm{SW}}(x, t)$ $=\Omega_{\mathrm{SW} 0} \sin k x \exp \left[-\left(t-t_{\mathrm{SW}}\right)^{2} / \sigma_{\mathrm{SW}}^{2}\right] . k=2 \pi / \lambda$ is the SW field wave number and $T=t_{\mathrm{SW}}-t_{\mathrm{TW}}=d / v_{z}$ is the characteristic STIRAP time with $d$ as the spatial separation between the centers of the two laser beams. $\Delta_{\mathrm{TW}}=\omega_{\mathrm{TW}}-\omega_{c b}\left(\Delta_{\mathrm{SW}}=\omega_{\mathrm{SW}}\right.$ $\left.-\omega_{c a}\right)$ is the single-photon detuning between the TW (SW) field and the corresponding transition. $\gamma_{a}\left(\gamma_{b}\right)$ is the spontaneous emission decay rate from $|c\rangle$ to $|a\rangle$ (from $|c\rangle$ to $|b\rangle$ ). 


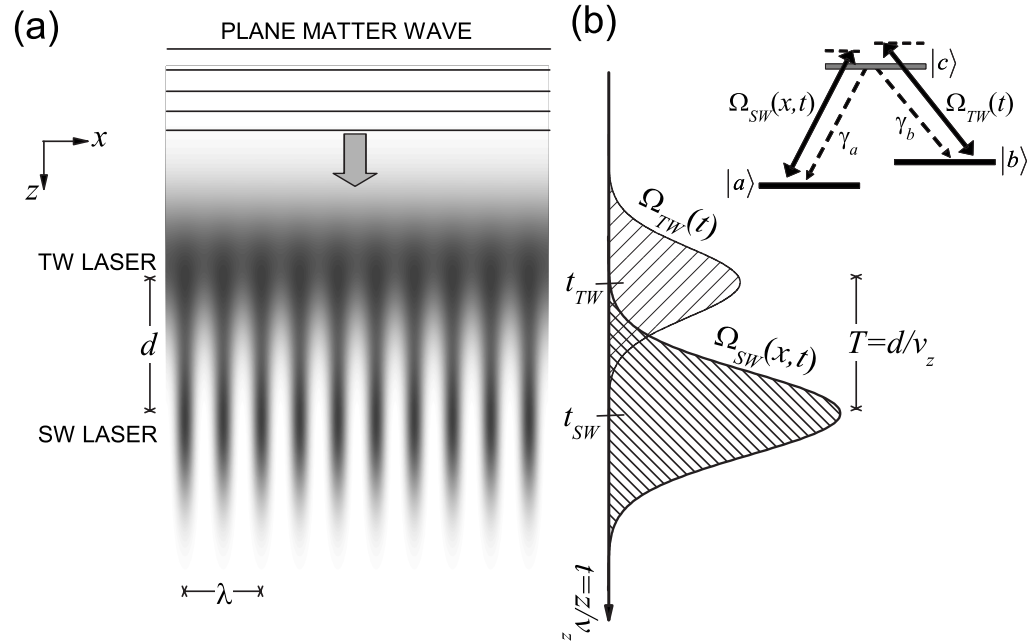

FIG. 1. (a) Schematics of the SLAP technique: a plane matter wave propagates consecutively through a TW and a partially overlapping SW laser field either in space (as shown here) or in time. (b) Three-level atomic system and Gaussian temporal profiles of the Rabi frequencies $\Omega_{\mathrm{TW}}(t)$ and $\Omega_{\mathrm{SW}}(x, t) . \gamma_{a}$ and $\gamma_{b}$ account for the spontaneous emission decay rates of the corresponding transition.

The spatial and the temporal variants of the SLAP technique are connected by the simple transformation $t=z / v_{z}$.

Under the two-photon resonance condition $\Delta_{\mathrm{TW}}=\Delta_{\mathrm{SW}}$, one of the position-dependent energy eigenstates of the $\Lambda$-type three-level system is the so-called dark state $|D(x, t)\rangle=\cos \theta(x, t)|a\rangle-\sin \theta(x, t)|b\rangle, \quad$ where $\quad \tan \theta(x, t)$ $=\Omega_{\mathrm{SW}}(x, t) / \Omega_{\mathrm{TW}}(t)$. STIRAP [16] consists in following this energy eigenstate from $\left|\psi_{\text {in }}\right\rangle=|a\rangle$ to $\left|\psi_{\text {out }}\right\rangle=|b\rangle$ smoothly changing $\theta$ from $0^{\circ}$ to $90^{\circ}$ by means of two partially overlapping laser fields as in the counterintuitive sequence of Fig. 1. To keep the system in the energy eigenstate, this process must be performed fulfilling the "global" adiabaticity condition [16],

$$
\Omega_{\mathrm{SW} 0}^{2} \sin ^{2} k x+\Omega_{\mathrm{TW} 0}^{2}>\left(\frac{A}{T}\right)^{2},
$$

where $A$ is a dimensionless constant that for optimal Gaussian profiles and overlapping times takes values around 10 [17].

In the SLAP technique, we assume that the matter wave has been initially prepared, by means of, e.g., optical pumping, into the internal state $|a\rangle$. Then, those atoms crossing the nodes of the SW remain in state $|a\rangle$, while those interacting with the TW and the SW fields through the STIRAP process are transferred to state $|b\rangle$. Therefore, an intense SW field should produce sharp peaks on the spatial population distribution of state $|a\rangle$ at its nodes. From Eq. (1) and assuming $A>T \Omega_{\mathrm{TW} 0}$, the full width at half maximum (FWHM) of these peaks is given by

$$
(\Delta x)_{\mathrm{SLAP}}=(\Delta x)_{\mathrm{CPT}} \frac{1}{2} \sqrt{\left(\frac{A}{T \Omega_{\mathrm{TW} 0}}\right)^{2}-1},
$$

where $(\Delta x)_{\mathrm{CPT}}=2 / k \sqrt{\mathcal{R}}$ with $\mathcal{R} \equiv \Omega_{\mathrm{SW} 0}^{2} / \Omega_{\mathrm{TW} 0}^{2}$ as the FWHM of the peaks in the Fabry-Pérot-type localization that would be attained by means of the CPT technique [11]. Therefore, for

$$
T \Omega_{\mathrm{TW} 0}=\frac{d}{v_{z}} \Omega_{\mathrm{TW} 0}>\frac{A}{\sqrt{5}}
$$

the superlocalization regime which we define by $(\Delta x)_{\text {SLAP }}$ $<(\Delta x)_{\mathrm{CPT}}$ is reached. Note that for $A=10$ corresponding to optimal parameter values [17], condition (3) reads $T \Omega_{\mathrm{TW}}$ $>4.5$.

Figure 2 shows numerical simulations of the stateselective localization process by integrating the corresponding density matrix equations for both the SLAP and the CPT techniques. In the CPT technique [11], subwavelength stateselective localization is obtained by reaching the steady state through an optical-pumping process to the dark state involving several cycles of laser excitation and spontaneous emis-

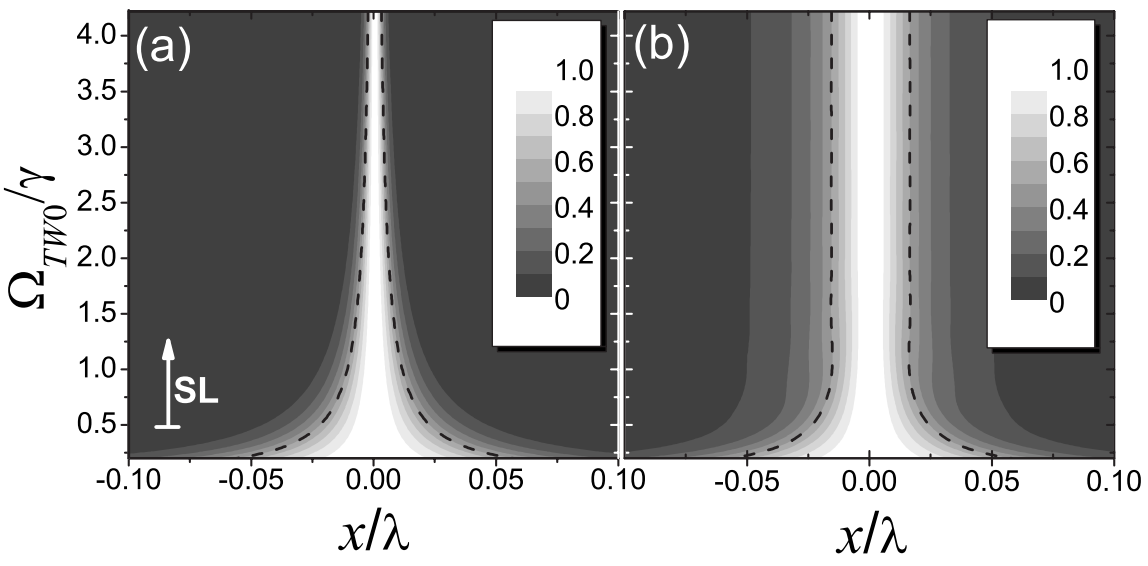

FIG. 2. Population distribution of state $|a\rangle$ after applying (a) the SLAP technique and (b) the CPT technique as a function of the amplitude of the TW Rabi frequency $\Omega_{\mathrm{TW} 0}$ for $\mathcal{R}=100, \gamma \sigma_{\mathrm{TW}}=\gamma \sigma_{\mathrm{SW}}$ $=5, \Delta_{\mathrm{TW}}=\Delta_{\mathrm{SW}}=0, \gamma T=10$ for the SLAP case, and $\gamma T=0$ for the CPT case. The horizontal separation between the dashed curves gives the FWHM of the corresponding localized structure. SL in (a) indicates the regime of superlocalization with $(\Delta x)_{\mathrm{SLAP}}$ $<(\Delta x)_{\mathrm{CPT}}$. 
sion. In the setup of Fig. 1, the CPT process corresponds to $T=0, \sigma_{\mathrm{TW}}=\sigma_{\mathrm{SW}}$, and $\gamma \sigma_{\mathrm{TW}} \gg 1$, where we have assumed, for simplicity, $\gamma_{a}=\gamma_{b}(\equiv \gamma)$. For $\mathcal{R}=100$ and the rest of parameters given in the figure caption, Fig. 2(a) shows that for $\Omega_{\mathrm{TW} 0}>0.45 \gamma$ superlocalization condition (3) is fulfilled and the SLAP technique yields better localization than the CPT technique, i.e., $(\Delta x)_{\mathrm{SLAP}}<(\Delta x)_{\mathrm{CPT}} \sim 0.032 \lambda$.

Note that we have considered here, for simplicity, a onedimensional (1D) SW field in the $|a\rangle-|b\rangle$ transition although the SLAP techniques apply also to higher dimensions and to any arbitrarily spatially structured field presenting intensity nodes.

\section{SLAP BASED NANOLITHOGRAPHY}

As a first implementation, we consider atom lithography based on substrates sensitive to the internal energy of metastable atoms [22]. For this purpose, we take a plane matter wave of metastable $\mathrm{Ne}^{*}$ whose initial internal level $=2 p^{5} 3 s\left({ }^{3} P_{0}\right)$ has an energy of $16.6 \mathrm{eV}$ and thus high potential for surface damage. In fact, $\mathrm{Ne}^{*}$ is a prime candidate for coherent manipulation since the STIRAP technique has been successfully reported with $\mathrm{Ne}^{*}[23]$ using the $\Lambda$ scheme $2 p^{5} 3 s\left({ }^{3} P_{0}\right) \leftrightarrow 2 p^{5} 3 p\left({ }^{3} P_{1}\right) \leftrightarrow 2 p^{5} 3 s\left({ }^{3} P_{2}\right)$ where the first and the last are long lived states [see Fig. 3(a)]. However, here we are interested in applying the SLAP technique such that, away from the nodes of the SW, the initial state is adiabatically transferred to a fast decaying state in order to remove the corresponding high internal energy. Thus we consider the open three-level $\Lambda$ scheme $2 p^{5} 3 s\left({ }^{3} P_{0}\right) \leftrightarrow 2 p^{5} 3 p\left({ }^{3} P_{1}\right)$ $\leftrightarrow 2 p^{5} 3 s\left({ }^{3} P_{1}\right)$ [depicted in Fig. 3(a)] with state $2 p^{5} 3 s\left({ }^{3} P_{1}\right)$ decaying to the ground state at a rate of $2 \pi \times 7.58$ $\times 10^{6} \mathrm{~s}^{-1}$. Figure 3(b) shows subwavelength atom localization in state $2 p^{5} 3 s\left({ }^{3} P_{0}\right)$ (solid curve) around a node of the $\mathrm{SW}$ (period of $308.2 \mathrm{~nm}$ ) after the application of the SLAP technique for $\mathcal{R}=400$ and the other parameters given in the figure caption. Note that although part of the population (dashed curve) is diabatically transferred to the high-energy state $2 p^{5} 3 s\left({ }^{3} P_{2}\right)$ with lifetime $\tau=14.73$ s [24], this population could be efficiently pumped to the ground state via $2 p^{5} 3 p\left({ }^{3} D_{2}\right)$ with an extra laser field. Thus, for realistic parameter values one would expect state-selective localization with a FWHM of only a few nanometers yielding high contrast peak energies of $80 \%$ [solid curve in Fig. 3(c)] in the absence of depumping of the $2 p^{5} 3 s\left({ }^{3} P_{2}\right)$ state and of nearly $100 \%$ [dashed curve in Fig. 3(c)] in the presence of the depumping.

As an important feature of the SLAP technique, the localized state $|a\rangle$ does not interact with the light fields at any time and therefore does not suffer from recoil induced broadening, which implies that the Raman-Nath approximation perfectly applies [18]. In this situation, the transversal velocity spread of the initial matter wave determines the ultimate resolution limit of the SLAP technique. Taking $\overline{\Delta v}_{x}$ as the rms transversal velocity spread, the limit $T \overline{\Delta v}_{x} \ll(\Delta x)_{\text {SLAP }}$ corresponds to $\overline{\Delta v}_{x} / v_{z} \ll(\Delta x)_{\mathrm{SLAP}} / d$. Thus, for typical parameters, $v_{z}=500 \mathrm{~m} / \mathrm{s}, \Delta v_{x}=5 \mathrm{~cm} / \mathrm{s}$, and $d=20 \mu \mathrm{m}$, localization down to single nanometer can be achieved. As given by the Heisenberg uncertainty principle, strong atom local- (a)

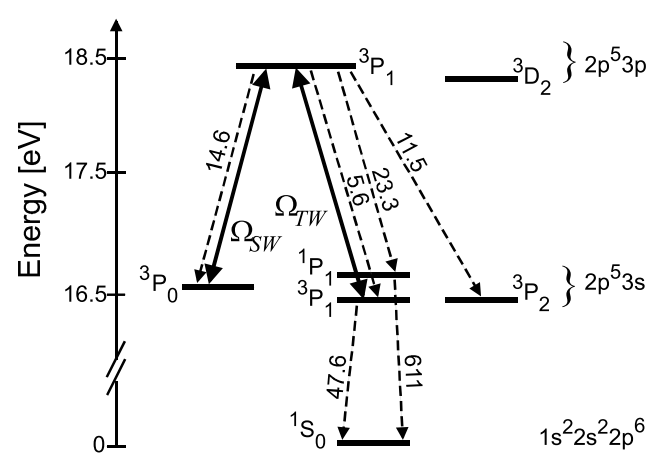

(b)

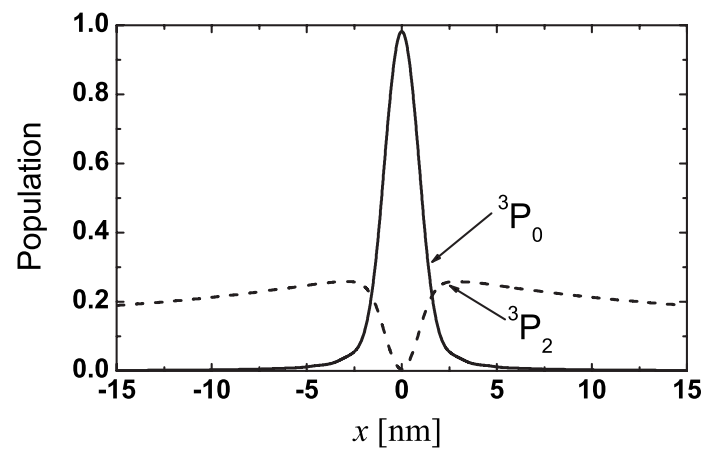

(c)

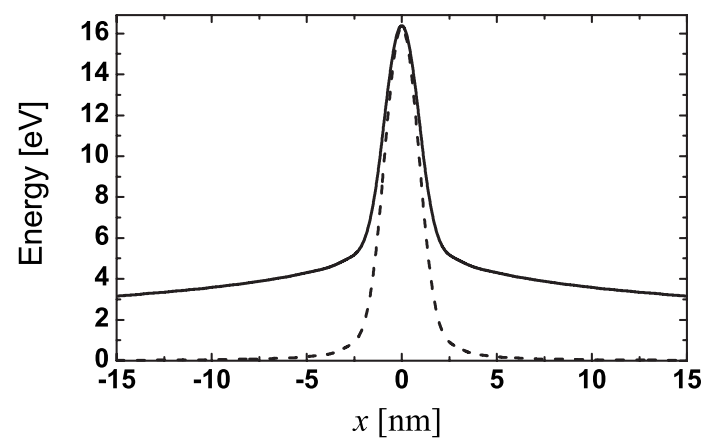

FIG. 3. SLAP technique for a $\mathrm{Ne}^{*}$ matter wave: (a) relevant energy levels and Einstein A coefficients (in units of $10^{6} \mathrm{~s}^{-1}$ ) for $\mathrm{Ne}^{*}$. The TW field at $\lambda_{\mathrm{TW}}=603.0 \mathrm{~nm}$ couples transition $2 p^{5} 3 s\left({ }^{3} P_{1}\right) \leftrightarrow 2 p^{5} 3 p\left({ }^{3} P_{1}\right)$, while the $\mathrm{SW}$ at $\lambda_{\mathrm{SW}}=616.4 \mathrm{~nm}$ couples $2 p^{5} 3 s\left({ }^{3} P_{0}\right) \leftrightarrow 2 p^{5} 3 p\left({ }^{3} P_{1}\right)$. (b) Final spatial population distribution around a node of the $\mathrm{SW}$ for states $2 p^{5} 3 s\left({ }^{3} P_{0}\right)$ (solid curve) and $2 p^{5} 3 s\left({ }^{3} P_{2}\right)$ (dashed curve). (c) Spatial distribution of the Ne matter wave internal energy after the SLAP technique around a node of the SW (solid curve) and after the depumping process of state $2 p^{5} 3 s\left({ }^{3} P_{2}\right)$ (dashed curve). Parameters: $v_{z}=500 \mathrm{~m} / \mathrm{s}, d=100 \mu \mathrm{m}$, $\sigma_{\mathrm{TW}}=\sigma_{\mathrm{SW}}=100 \mathrm{~ns}, \mathcal{R}=400, \Omega_{\mathrm{TW} 0}=2 \pi \times 1.6 \times 10^{7} \mathrm{~s}^{-1}$, and $\Delta_{\mathrm{TW}}$ $=\Delta_{\mathrm{SW}}=0$.

ization should also result in the appearance of high momentum components [11]. For the results shown in Fig. 3(b), we have verified that the highest momentum components do not have time enough to smear out the localized structure until the end of the SW where the substrate is placed.

\section{COHERENT PATTERNING OF A BEC BASED ON SLAP}

As a second implementation, we now focus on a trapped $\mathrm{BEC}$ of ${ }^{87} \mathrm{Rb}$ to show the feasibility to generate narrow struc- 


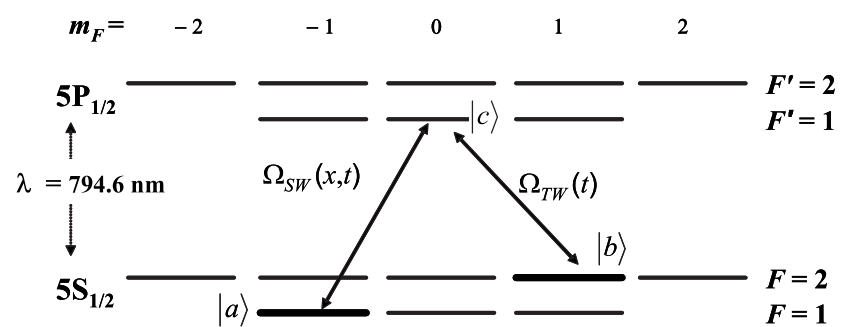

FIG. 4. Hyperfine structure of the D1 transition line of ${ }^{87} \mathrm{Rb}$ with the couplings $\Omega_{\mathrm{SW}}(x, t)$ and $\Omega_{\mathrm{TW}}(t)$ defining the $\Lambda$ scheme. The broader lines correspond to the two condensed trapped states $|a\rangle=\left|F=1, m_{F}=-1\right\rangle$ and $|b\rangle=\left|F=2, m_{F}=1\right\rangle$.

tures in the condensate by means of the SLAP technique. The $\Lambda$-type three-level configuration under study is depicted in Fig. 4. We consider a zero temperature two-species ${ }^{87} \mathrm{Rb}$ BEC, $|a\rangle=\left|F=1, m_{F}=-1\right\rangle$ and $|b\rangle=\left|F=2, m_{F}=1\right\rangle$, confined in a one-dimensional geometry. The description of the system is performed within the 1D coupled Gross-Pitaevskii equations,

$$
\begin{aligned}
& i \hbar \frac{d \psi_{a}}{d t}= {\left[-\frac{\hbar^{2}}{2 m} \Delta+V_{a}(x)+g_{a a}\left|\psi_{a}\right|^{2}+g_{a b}\left|\psi_{b}\right|^{2}\right] \psi_{a} } \\
&+\frac{1}{2} \hbar \Omega_{\mathrm{SW}}(x, t) \psi_{c}, \\
& i \hbar \frac{d \psi_{b}}{d t}= {\left[-\frac{\hbar^{2}}{2 m} \Delta+V_{b}(x)+g_{b b}\left|\psi_{b}\right|^{2}+g_{a b}\left|\psi_{a}\right|^{2}\right] \psi_{b} } \\
&+\frac{1}{2} \hbar \Omega_{\mathrm{TW}}(t) \psi_{c}+\hbar\left(\Delta_{\mathrm{SW}}-\Delta_{\mathrm{TW}}\right) \psi_{b}, \\
& i \hbar \frac{d \psi_{c}}{d t}=\frac{1}{2} \hbar \Omega_{\mathrm{SW}}(x, t) \psi_{a}+\frac{1}{2} \hbar \Omega_{\mathrm{TW}}(t) \psi_{b}-i \frac{\Gamma}{2} \psi_{c}+\hbar \Delta_{\mathrm{SW}} \psi_{c},
\end{aligned}
$$

where the effective $1 \mathrm{D}$ nonlinearity is given by $g_{i j}=2 \hbar a_{i j} \omega_{t}$, $i, j=a, b$ with $a_{i j}$ as the interspecies $(i \neq j)$ and intraspecies $(i=j) s$-wave scattering lengths and $\omega_{t}$ as the transverse trapping frequency. In ${ }^{87} \mathrm{Rb}$ the scattering lengths are known to be in the proportion $a_{a a}: a_{a b}: a_{b b}=1.03: 1: 0.97$ with the average of the three being 55(3) $\AA$ [25]. Since the magnetic moments of the two trapped components are the same to first order, magnetic trapping as well as optical trapping is possible with equal potentials for both components. The axial trapping potential reads $V_{a}(x)=V_{b}(x)=m \omega_{x}^{2} x^{2} / 2$, with $m$ as the ${ }^{87} \mathrm{Rb}$ mass and $\omega_{x}$ as the axial trapping frequency. State $|c\rangle=\left|F^{\prime}=1, m_{F}=0\right\rangle$ is not trapped and the excited atoms are assumed to escape from the BEC at a rate $\Gamma=2 \pi \times 5.41$ $\times 10^{6} \mathrm{~s}^{-1}$.

To show the time evolution of the system during the SLAP process, we have numerically solved Eqs. (4)-(6) for a BEC of $5 \times 10^{4}$ atoms. Figures $5(\mathrm{a})$ and $5(\mathrm{~b})$ show the mixing angle $\theta$ at one of the SW antinodes and the contour plot of the density distribution of atoms in state $|a\rangle$, respectively, as a function of time and for the parameters given in the figure caption. As expected, component $|a\rangle$ develops extremely narrow structures at the nodes of the SW whose
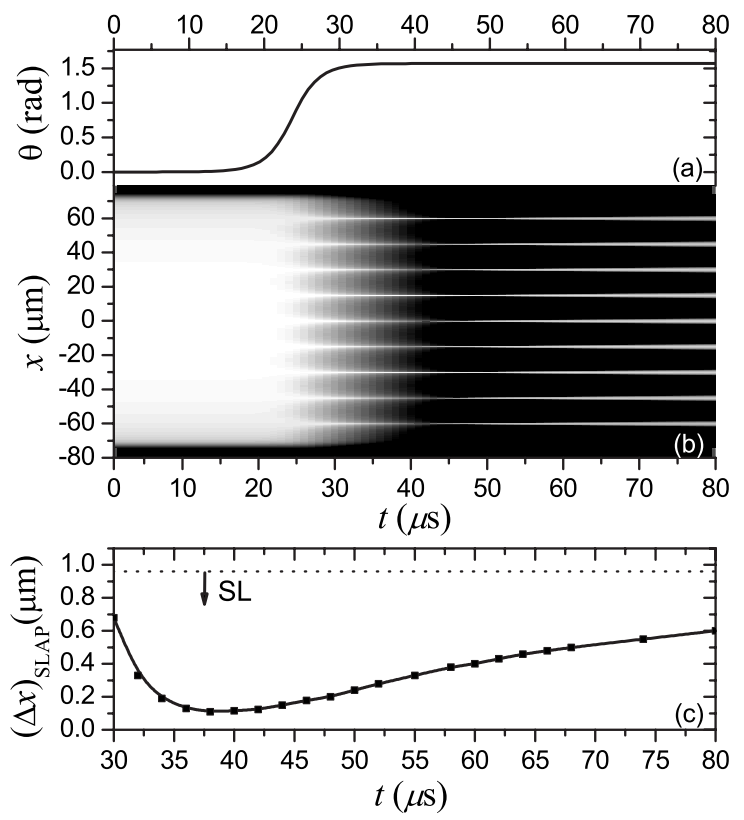

FIG. 5. SLAP technique for a ${ }^{87} \mathrm{Rb}$ BEC: (a) time evolution of the mixing angle $\theta$ at one of the SW antinodes. (b) Contour plot of the density distribution of atoms in state $|a\rangle$ as a function of time during the SLAP technique for a SW with $15 \mu \mathrm{m}$ period, $\mathcal{R}=100$, $\Omega_{\mathrm{TW} 0}=2 \pi \times 10 \times 10^{6} \mathrm{~s}^{-1}, \quad \sigma_{\mathrm{TW}}=\sigma_{\mathrm{SW}}=8 \mu \mathrm{s}, \quad t_{\mathrm{TW}}=22 \mu \mathrm{s}, \quad t_{\mathrm{SW}}$ $=36 \mu \mathrm{s}, \Delta_{\mathrm{TW}}=\Delta_{\mathrm{SW}}=0, \omega_{x}=2 \pi \times 14 \mathrm{~s}^{-1}$, and $\omega_{t}=2 \pi \times 715 \mathrm{~s}^{-1}$. (c) Time evolution of the FWHM of the central localized structure.

width is much smaller than its spatial period. For demonstration, we have chosen a large spatial period of $15 \mu \mathrm{m}$ but arbitrary periods down to $\lambda_{\mathrm{SW}} / 2$ with a corresponding localization down to the nanometer scale could be achieved by changing the wave number $k$ of the standing wave. Figure 5(c) shows the time evolution of the FWHM around one node. The minimal width of the localized structures coincides approximately with the time at which the TW field is switched off, i.e., at $\theta=\pi / 2$. We have calculated the transverse momentum spread $\Delta p_{x}$ for this time, obtaining a beam quality factor [26] $M^{2}=(2 / \hbar) \Delta x \Delta p_{x} \approx 0.6$ which is below the Heisenberg limit due to the nonlinearities of the twocomponent trapped BEC partially compensating for diffraction.

\section{CONCLUSIONS AND PERSPECTIVES}

In conclusion, we have proposed the SLAP technique for state-selective localization and patterning of atomic matter waves. We have shown that a superlocalization regime beating the previously introduced CPT localization technique [11] can be reached and analytic expressions for the necessary conditions have been derived. We have discussed the use of the SLAP technique for nanolithograhy with a $\mathrm{Ne}^{*}$ matter wave showing the possibility to imprint high contrast patterns with narrow structures whose FWHM approaches the single nanometer scale. This lithographic technique is applicable to all atomic systems with a high-energy dark state formed by the combination of a state fast decaying to the ground and a metastable one. Coherent patterning of a 
two-component ${ }^{87} \mathrm{Rb}$ BEC in the superlocalization regime has been studied in detail as a second example showing that it is possible to overpass the Heisenberg limit.

Since localization occurs at the nodes of one of the involved laser fields, more evolved patterning schemes can be realized by extending the present $1 \mathrm{D}$ configuration to higher dimensions by applying two-dimensional (2D) and threedimensional (3D) SW configurations. Even more complex structures, such as the intensity nodes of higher-order Laguerre-Gauss modes or the light fields of custom-made micro-optical elements [27], could be considered for this technique. Following the presented SLAP technique for coherent patterning of a BEC, one could consider its application to produce a collection of parallel, coherent, and extremely collimated (pulsed) atom lasers [28] or, by observing the corresponding near field diffraction pattern, to investigate the matter wave analog of the optical Talbot effect [29]. Fi- nally, the SLAP technique could also be applied to address and detect individual sites in optical lattices by an appropriate choice between the spatial period of the optical lattice and the wavelength of the SW field used in this technique.

\section{ACKNOWLEDGMENTS}

We acknowledge support by the Spanish Ministry of Education and Science under Contracts No. FIS2005-01497, No. FIS2005-01369, No. FIS2008-02425, No. HA2005-0002, No. HD2008-0078, and No. CSD2006-00019, by the Catalan Government under Contract No. SGR2005-00358, by the ESF and DFG under the project CIGMA, by the European Commission within the RTN Atom Chips and the IP SCALA, by the DAAD under Contract No. 0804149, and by the NIST under Award No. 60NANB5D120.
[1] For a review see I. Bloch, Nat. Phys. 1, 23 (2005); M. Lewenstein et al., Adv. Phys. 56, 243 (2007); I. Bloch, J. Dalibard, and W. Zwerger, Rev. Mod. Phys. 80, 885 (2008).

[2] R. Scheunemann, F. S. Cataliotti, T. W. Hansch, and M. Weitz, Phys. Rev. A 62, 051801(R) (2000); M. Saffman, Opt. Lett. 29, 1016 (2004); D. Schrader, I. Dotsenko, M. Khudaverdyan, Y. Miroshnychenko, A. Rauschenbeutel, and D. Meschede, Phys. Rev. Lett. 93, 150501 (2004); J. Cho, ibid. 99, 020502 (2007); M. Karski, L. Forster, J. M. Choi, W. Alt, A. Widera, and D. Meschede, ibid. 102, 053001 (2009); K. D. Nelson, X. Li, and D. Weiss, Nat. Phys. 3, 556 (2007).

[3] K. Staliunas, S. Longhi, and G. J. de Valcarcel, Phys. Rev. Lett. 89, 210406 (2002); M. Modugno, C. Tozzo, and F. Dalfovo, Phys. Rev. A 74, 061601(R) (2006).

[4] For a review on matter wave lithography see C. A. Mack, Fundamental Principles of Optical Lithography: The Science of Microfabrication (Chichester, West Sussex, England, 2007); M. K. Oberthaler and T. Pfau, J. Phys.: Condens. Matter 15, R233 (2003); J. H. Thywissen and M. Prentiss, New J. Phys. 7, 47 (2005); D. Meschede and H. Metcalf, J. Phys. D 36, R17 (2003).

[5] U. Drodofsky, J. Stuhler, B. Brezger, Th. Schulze, M. Drewsen, T. Pfau, and J. Mlynek, Microelectron. Eng. 35, 285 (1997); R. E. Behringer, V. Natarajan, G. Timp, and D. M. Tennant, J. Vac. Sci. Technol. B 14, 4072 (1996); Th. Schulze, B. Brezger, R. Mertens, M. Pivk, T. Pfau, and J. Mlynek, Appl. Phys. B: Lasers Opt. 70, 671 (2000).

[6] R. Abfalterer, C. Keller, S. Bernet, M. K. Oberthaler, J. Schmiedmayer, and A. Zeilinger, Phys. Rev. A 56, R4365 (1997); K. S. Johnson, J. H. Thywissen, N. H. Dekker, K. K. Berggren, A. P. Chu, R. Younkin, and M. Prentiss, Science 280, 1583 (1998).

[7] P. R. Hemmer, A. Muthukrishnan, M. O. Scully, and M. S. Zubairy, Phys. Rev. Lett. 96, 163603 (2006); Q. Sun, P. R. Hemmer, and M. S. Zubairy, Phys. Rev. A 75, 065803 (2007).

[8] M. Holland, S. Marksteiner, P. Marte, and P. Zoller, Phys. Rev. Lett. 76, 3683 (1996).

[9] E. Paspalakis and P. L. Knight, Phys. Rev. A 63, 065802
(2001)

[10] M. Sahrai, H. Tajalli, K. T. Kapale, and M. S. Zubairy, Phys. Rev. A 72, 013820 (2005).

[11] G. S. Agarwal and K. T. Kapale, J. Phys. B 39, 3437 (2006).

[12] M. Kiffner, J. Evers, and M. S. Zubairy, Phys. Rev. Lett. 100, 073602 (2008).

[13] A. V. Gorshkov, L. Jiang, M. Greiner, P. Zoller, and M. D. Lukin, Phys. Rev. Lett. 100, 093005 (2008).

[14] S. E. Harris, Phys. Today 50(7), 36 (1997); E. Arimondo, in Progress in Optics, edited by E. Wolf (Elseveir Science, Amsterdam, 1996), Vol. XXXV, p. 257; M. O. Scully and M. S. Zubairy, Quantum Optics (Cambridge University Press, England, 1997); J. P. Marangos, J. Mod. Opt. 45, 471 (1998); J. Mompart and R. Corbalán, J. Opt. B: Quantum Semiclassical Opt. 2, R7 (2000); F. Silva, J. Mompart, V. Ahufinger, and R. Corbalan, Phys. Rev. A 64, 033802 (2001).

[15] H. Li, V. A. Sautenkov, M. M. Kash, A. V. Sokolov, G. R. Welch, Y. V. Rostovtsev, M. S. Zubairy, and M. O. Scully, Phys. Rev. A 78, 013803 (2008).

[16] K. Bergmann, H. Theuer, and B. W. Shore, Rev. Mod. Phys. 70, 1003 (1998).

[17] J. R. Kuklinski, U. Gaubatz, F. T. Hioe, and K. Bergmann, Phys. Rev. A 40, 6741 (1989).

[18] See Pierre Meystre, Atom Optics (Springer, New York, 2001), and reference therein.

[19] A. Vardi, D. Abrashkevich, E. Frishman, and M. Shapiro, J. Chem. Phys. 107, 6166 (1997); P. S. Julienne, K. Burnett, Y. B. Band, and W. C. Stwalley, Phys. Rev. A 58, R797 (1998); M. Mackie, R. Kowalski, and J. Javanainen, Phys. Rev. Lett. 84, 3803 (2000); J. J. Hope, M. K. Olsen, and L. I. Plimak, Phys. Rev. A 63, 043603 (2001); P. D. Drummond, K. V. Kheruntsyan, D. J. Heinzen, and R. H. Wynar, ibid. 65 , 063619 (2002); H. Y. Ling, H. Pu, and B. Seaman, Phys. Rev. Lett. 93, 250403 (2004); J. Cheng, S. Han, and Y. J. Yan, Phys. Rev. A 73, 035601 (2006).

[20] K. Winkler, G. Thalhammer, M. Theis, H. Ritsch, R. Grimm, and J. H. Denschlag, Phys. Rev. Lett. 95, 063202 (2005).

[21] K. C. Wright, L. S. Leslie, and N. P. Bigelow, Phys. Rev. A 
77, 041601(R) (2008).

[22] P. Engels, S. Salewski, H. Levsen, K. Sengstock, and W. Ertmer, Appl. Phys. B: Lasers Opt. 69, 407 (1999).

[23] J. Martin, B. W. Shore, and K. Bergmann, Phys. Rev. A 54, 1556 (1996).

[24] M. Zinner, P. Spoden, T. Kraemer, G. Birkl, and W. Ertmer, Phys. Rev. A 67, 010501(R) (2003).

[25] J. M. Vogels, C. C. Tsai, R. S. Freeland, S. J. J. M. F. Kokkelmans, B. J. Verhaar, and D. J. Heinzen, Phys. Rev. A 56, R1067 (1997); M. R. Matthews, D. S. Hall, D. S. Jin, J. R. Ensher, C. E. Wieman, E. A. Cornell, F. Dalfovo, C. Minniti, and S. Stringari, Phys. Rev. Lett. 81, 243 (1998).

[26] J. F. Riou, W. Guerin, Y. Le Coq, M. Fauquembergue, V. Josse, P. Bouyer, and A. Aspect, Phys. Rev. Lett. 96, 070404 (2006); A. E. Siegman, IEEE J. Quantum Electron. 27, 1146 (1991); M. Jeppesen, J. Dugue, G. R. Dennis, M. T. Johnsson, C. Figl,
N. P. Robins, and J. D. Close, Phys. Rev. A 77, 063618 (2008); F. Impens, ibid. 77, 013619 (2008).

[27] G. Birkl, F. B. J. Buchremer, R. Dumke, and W. Ertmer, Opt. Commun. 191, 67 (2001); F. B. J. Buchkremer, R. Dumke, M. Volk, T. Muther, G. Birkl, and W. Ertmer, Laser Phys. 12, 736 (2002).

[28] M. O. Mewes, M. R. Andrews, D. M. Kurn, D. S. Durfee, C. G. Townsend, and W. Ketterle, Phys. Rev. Lett. 78, 582 (1997); H. M. Wiseman, Phys. Rev. A 56, 2068 (1997); B. P. Anderson and M. A. Kasevich, Science 282, 1686 (1998); I. Bloch, T. W. Hänsch, and T. Esslinger, Phys. Rev. Lett. 82, 3008 (1999); E. W. Hagley, L. Deng, M. Kozuma, J. Wen, K. Helmerson, S. L. Rolston, and W. D. Phillips, Science 283, 1706 (1999).

[29] A. Turlapov, A. Tonyushkin, and T. Sleator, Phys. Rev. A 71, 043612 (2005). 